\title{
PD-L1 and MHC-I expression in 19 human tumor cell lines and modulation by interferon-gamma treatment
}

\author{
Italia Grenga ${ }^{1 *}$, Renee N Donahue ${ }^{2}$, Lauren Lepone ${ }^{1}$, Jessa Bame${ }^{1}$, Jeffrey Schlom¹, Benedetto Farsaci ${ }^{1}$ \\ From Society for Immunotherapy of Cancer 29th Annual Meeting \\ National Harbor, MD, USA. 6-9 November 2014
}

\section{Background}

The aim of this study was to analyze the expression of PD-L1 and MHC-I in 19 human tumor cell lines and changes after interferon gamma (IFN- $\gamma$ ) treatment, in order to evaluate the potentiality of combining anti-PDL1 antibody with other immunotherapies.

\section{Methods}

Nineteen human tumor cell lines were cultured according with ATCC guidelines: 5 colon (Caco-2, SW620, SW480, Colo-205 and HT-29), 4 ovarian (OV-17, OVCAR-3, ES-2, SKOV-3), 3 breast (MDA-MB-231, MCF-7, ZR-75), 3 lung (H441, H1703, H460), 2 prostate (LnCap and PC-3), and 2 pancreatic (CFPAC-1 and ASPC-1). Cells were analyzed by flow-cytometry for PD-L1 (clone 29E.2A3) and MHC-I expression. The surface expression of PD-L1 was considered as low, medium, or high based on the percentage of positive cells (80\%, respectively). Cells were also analyzed for PD-L1 mRNA expression by RT-PCR. Experiments were performed with or without IFN- $\gamma$ pre-treatment (10 ng/ml, 24 hours).

\section{Results}

The expression of PD-L1 was as follows. Low: 4/5 colon (SW620, SW480, Colo-205 and HT-29), 1/4 ovarian (OVCAR-3), $2 / 3$ breast (ZR-75, MCF-7), and $1 / 2$ pancreatic (ASPC-1). Medium: 1/5 colon (Caco-2), 2/4 ovarian (OV-17, SKOV-3), $2 / 3$ lung (H460, H1703), and 1/2 prostate (LnCap). High: 1/4 ovarian (ES-2), 1/3 lung (H441), and $1 / 2$ prostate (PC-3), 1/3 breast (MDA-MB-231), and $1 / 2$ pancreatic (ASPC-1). After IFN- $\gamma$ pre-treatment, 14/19 cell lines showed a $>50 \%$ increase of PD-L1 and 14/19 a
$>50 \%$ increase of MHC-I (either percentage positive or MFI). In 13/19 cell lines both markers increased. IFN- $\gamma$ pre-treatment caused an increase $>100 \%$ of PD-L1 mRNA expression in 14/19 cell lines. CFPAC-1 (pancreatic) showed an increase of surface PD-L1 without mRNA change; on the opposite, H1703 (lung) showed mRNA increase without changes in surface expression.

\section{Conclusions}

Tumor cells express different percentage of PD-L1 and MHC-I in their surface. In most of the cells analyzed, both molecules are increased by exposure to IFN- $\gamma$. Based on these observations, immunotherapies aiming to increase IFN- $\gamma$ in the tumor microenvironment, such as therapeutic vaccines or $\mathrm{T}$ cell adoptive transfer, can facilitate immune recognition of tumor cells by an increase of MHC-I on the surface of tumor cells. On the other hand, the increased PD-L1 expression in the tumor can be an ideal target for anti-PD-L1 antibody treatment.

\section{Authors' details \\ 'Laboratory of Tumor Immunology and Biology, CCR, NCl, NIH, Bethesda,} MD, USA. ${ }^{2} \mathrm{NCI} / \mathrm{CCR} / \mathrm{LTIB}$, Bethesda, MD, USA.

Published: 6 November 2014

doi:10.1186/2051-1426-2-S3-P102

Cite this article as: Grenga et al:: PD-L1 and MHC-I expression in 19 human tumor cell lines and modulation by interferon-gamma treatment. Journal for ImmunoTherapy of Cancer 2014 2(Suppl 3):P102. 